\title{
Protective effects of tyndallized Lactobacillus acidophilus IDCC 3302 against UVB-induced photodamage to epidermal keratinocytes cells
}

\author{
A-RANG IM ${ }^{1,2}$, BYEONGHUN LEE ${ }^{2}$, DAE-JUNG KANG ${ }^{2}$ and SUNGWOOK CHAE ${ }^{1,3}$ \\ ${ }^{1}$ Herbal Medicine Research Division, Korea Institute of Oriental Medicine, Daejeon 34054; \\ ${ }^{2}$ Bioprocess Engineering Team, Research Laboratories, ILDONG Pharmaceutical Co., Ltd., Hwaseong, Gyeonggi 18449; \\ ${ }^{3}$ University of Science and Technology (UST), Korean Medicine Life Science, Daejeon 34113, Republic of Korea
}

Received February 26, 2018; Accepted April 8, 2019

DOI: $10.3892 /$ ijmm.2019.4161

\begin{abstract}
Photoaging is a consequence of chronic exposure to ultraviolet (UV) radiation and results in skin damage. In this study, whether tyndallizate of the probiotic bacterium Lactobacillus acidophilus IDCC 3302 (ACT 3302) can protect against UVB-induced photodamage to the skin was investigated. For this, HaCaT keratinocytes were used as a model for skin photoaging. HaCaT cells were treated with ACT 3302 prior to UVB exposure and skin hydration factors and matrix metalloproteinase (MMP)-1, MMP-2, and MMP-9 levels in the culture supernatant were evaluated by ELISA. The protective effects of ACT 3302 against UVB-induced oxidative stress in HaCaT cells was also assessed by measuring superoxide dismutase and catalase activity and detecting the expression of pro-inflammatory cytokine-encoding genes and mitogen-activated protein kinase (MAPK) signaling components by reverse transcription-quantitative polymerase chain reaction and western blotting, respectively. UVB exposure increased MMP expression and MAPK activation; these changes were attenuated by pretreatment with ACT 3302. Treatment with ACT 3302 prior to UVB exposure also attenuated inflammation. These results demonstrate that tyndallized ACT 3302 can mitigate photodamage to the skin induced by UVB radiation through the suppression of MMPs and could therefore be used clinically to prevent wrinkle formation.
\end{abstract}

\section{Introduction}

Extrinsic aging is caused by environmental oxidative factors that can be primarily attributed to exposure to ultraviolet (UV)

Correspondence to: Dr Sungwook Chae, Herbal Medicine Research Division, Korea Institute of Oriental Medicine, 1672 Yuseong-daero, Yuseong-gu, Daejeon 34054, Republic of Korea

E-mail: kendall@kiom.re.kr

Key words: Lactobacillus acidophilus IDCC 3302 tyndallizate, matrix metalloproteinase, mitogen-activated protein kinase, photodamage, skin hydration, ultraviolet $\mathrm{B}$ radiation radiation (1). A variety of environmental stresses including UV light can damage sun-exposed areas of the skin including the face and neck, and accelerate wrinkle formation (2), which is a cumulative process that depends on the frequency, duration, and intensity of UV exposure and the degree of natural protection offered by skin pigmentation (3). In addition to wrinkling, skin aging manifests as solar elastosis and pigment irregularities (4).

UVB radiation alters the thickness of the stratum corneum of the epidermis and consequently, the permeability barrier, leading to increased transepidermal water loss (TEWL) (5). Several factors control skin moisturization and elasticity, including the content of hyaluronan (HA), an extracellular matrix (ECM) component and transforming growth factor (TGF)- $\beta$, in addition to regulators of the expression of ECM macromolecules (6). Collagen has been used as a functional ingredient in skin products based on its efficacy in moisturizing and enhancing elasticity. Furthermore, involucrin and filaggrin are major proteins that serve an important role in the formation of the epidermal skin barrier (7). Changes to the dermal ECM during photoaging involve the abnormal production of matrix proteins by dermal fibroblasts and increased the activity of matrix-degrading enzymes such as matrix metalloproteinases (MMPs) by resident skin cells and infiltrating inflammatory cells (8). Also involved is decreased procollagen synthesis, resulting in the loss of skin elasticity, which leads to wrinkle formation (9).

Reactive oxygen species (ROS) produced in the skin upon UV irradiation cause oxidative damage to the skin (10) by mediating impaired cellular and ECM functions $(11,12)$. UVB radiation induces cellular damage, leading to activation of ROS-sensitive signaling pathways including the mitogenactivated protein kinase (MAPK) pathway (13), which mediates inflammatory cytokine production (14). Accordingly, antioxidants can protect the skin from UVB radiation-induced skin damage (15).

Probiotics have been widely used to regulate intestinal health and treat medical conditions including allergic diseases and atopic dermatitis, as well as to prevent dental caries and respiratory infections (16). The most common microorganisms used as probiotic bacteria, which include Lactobacillus, 
Bifidobacterium and Streptococcus, mainly affect health and improve gastrointestinal tract homeostasis in the host by modulating the balance in the gut environment (17). Tyndallized probiotics, which are processed in their culture media, contain bacterial walls; moreover, the products released during their cell death have been demonstrated to positively affect human health and maintain their immunological effect on the gut barrier (18). It has been reported that probiotic bacteria may be highly effective in protecting the skin from photoaging, as oral supplementation prior to UVB exposure was demonstrated to prevent TEWL, increase epidermal thickness, and alleviate damage to tight junction structures and the basement membrane in a mouse model $(19,20)$. The probiotic gram-positive bacterium Lactobacillus acidophilus inhabits the intestines and serves an important role in the maintenance of gut health; however, it is not known whether it can protect against photoaging induced by UV radiation.

This was addressed in the present study using $\mathrm{HaCaT}$ keratinocytes treated with Lactobacillus acidophilus IDCC 3302 tyndallizate (ACT 3302) prior to UVB exposure. Lactobacillus acidophilus IDCC 3302 (GenBank accession number KP325412.1) was originally isolated from the feces of a Korean breast-fed infant after obtaining oral consent from the parents. The materials used in the present experiments were not directly separated from human feces, but were commercially available materials produced by Il-dong Pharmaceutical. The results of the present study indicated that ACT 3302 treatment can prevent photodamage to the skin by inhibiting MMP activation through the modulation of MAPK signaling.

\section{Materials and methods}

Materials. Dulbecco's modified Eagle's medium (DMEM) and fetal bovine serum (FBS) were purchased from Gibco (Thermo Fisher Scientific, Inc., Waltham, MA, USA). TheCellTiter AQueous One Solution CellProliferation Assaykit and 3-(4,5-dimethylthiazol-2-yl)-5-(3-carboxymethoxyphenyl) -2-(4-sulfophenyl)-2H-tetrazolium (MTS) were purchased from Promega Corporation (Madison, WI, USA). Human HA and MMP-1, MMP-2 and MMP-9 ELISA kits were obtained from R\&D Systems, Inc., (Minneapolis, MN, USA). Antibodies targeting MAPK kinase (MEK), phosphorylated (p)MEK, extracellular signal-regulated kinase 1 and 2 (ERK1/2), pERK1/2, p38, pp38, c-Jun N-terminal kinase (JNK), pJNK, procollagen, and $\beta$-actin were purchased from Cell Signaling Technology, Inc., (Danvers, MA, USA). Secondary antibodies were purchased from Santa Cruz Biotechnology, Inc., (Santa Cruz, CA, USA).

Tyndallization process. Bacterial cells were anaerobically cultured in yeast extract-based medium at $37^{\circ} \mathrm{C}$ overnight and then centrifuged at $12,500 \mathrm{x}$ for $1 \mathrm{~h}$ at room temperature using a continuous filter (Alfa Laval, Lund, Sweden). Thereafter, the pellets and the supernatant were concentrated 5 times using a vacuum concentrator (Dong Yang Machine Industry, Seoul, Korea) under reduced pressure at $80^{\circ} \mathrm{C}$ and then mixed with dry-sterilized cornstarch powder. The cornstarch was not effective on wrinkle reduction. The mix was then frozen at $-45^{\circ} \mathrm{C}$ and lyophilized to obtain ACT 3302 powder.
Total bacterial cell numbers. ACT 3302 cell samples were diluted $10^{3}$-fold in PBS and cell particles in 25 small squares were counted using a Neubauer counting chamber (Paul Marienfeld GmbH \& Co., KG, Lauda-Königshofen, Germany), with $2.5 \times 10^{-4}-\mathrm{mm}^{3}$ squares, at a magnification of $\mathrm{x} 400$. The number of cells was calculated as follows: Bacterial cell number per $\mathrm{ml}=$ average number of counted cells per square $\mathrm{x} 4,000$ squares per $\mathrm{mm}^{3} \times 10^{3} \mathrm{~mm}^{3}$ per $\mathrm{ml} \times 10^{3}$ (dilution).

Cell culture and UVB irradiation. HaCaT cells (cell lines service, 300493)-an immortalized, non-tumorigenic human keratinocyte cell line were maintained in DMEM supplemented with $10 \%$ FBS and $1 \%$ antibiotics (Penicillin-Streptomycin; Gibco; Thermo Fisher Scientific, Inc.) at $37^{\circ} \mathrm{C}$ in a humidified incubator with $5 \% \mathrm{CO}_{2}$. Cells were seeded $\left(1 \times 10^{4}\right)$, allowed to adhere for $24 \mathrm{~h}$ and treated with various concentrations $\left(1 \times 10^{5}\right.$, $1 \times 10^{6}, 1 \times 10^{7}$ and $1 \times 10^{8}$ ) of ACT 3302 prior to UVB irradiation at a dose of $20 \mathrm{~mJ} / \mathrm{cm}^{2}$. Normal cells received no ACT 3302 and were not exposed to UVB radiation. Vehicle cells received no ACT 3302, but were exposed to UVB radiation.

Cell viability assay. HaCaT cells $\left(1 \times 10^{4}\right)$ were seeded in 96-well culture plates and exposed to $20 \mathrm{~mJ} / \mathrm{cm}^{2} \mathrm{UVB}$ in the presence or absence of ACT 3302 for $24 \mathrm{~h}$. The cell culture medium was replaced with PBS prior to UVB treatment and cell viability was assessed immediately following this by incubating cells with MTS (Promega Corporation) containing serum free medium for $1 \mathrm{~h}$. MTS containing serum free medium was measured according to the manufacturer's protocol; specifically, sample absorbance was measured at $490 \mathrm{~nm}$ using a microplate fluorimeter (Molecular Devices, LLC, Sunnyvale, CA, USA).

Determination of $H A, M M P-1, M M P-2$ and $M M P-9$ secretion by ELISA. HA (cat. no. DHYAL0), MMP-1 (cat. no. DMP100), MMP-2 (cat. no. MMP200) and MMP-9 (cat. no. DMP900) levels in HaCaT cell culture supernatant $\left(5 \times 10^{4}\right)$, following UVB irradiation, were determined using ELISA kits according to the manufacturer's protocol. Briefly, the cells were seeded in 96-well plates and treated with ACT 3302. After cells were treated with ACT 3302 for $24 \mathrm{~h}$, the medium was removed and then replaced with new medium for ELISA analysis. After UVB irradiation, the culture supernatant was collected and centrifuged at 18,928 $\mathrm{x} \mathrm{g}$ for 5 min at $4^{\circ} \mathrm{C}$ and HA, MMP-1, MMP-2, and MMP-9 levels were quantified colorimetrically.

Antioxidant enzyme activities. Total protein was extracted from $\mathrm{HaCaT}$ cells, which were homogenized in cold lysis buffer, superoxide dismutase (SOD) and catalase (CAT) activities were measured using colorimetric assay kits (Cayman Chemical, Ann Arbor, MI, USA) according to the manufacturer's protocol. SOD and CAT activities were determined by measuring the absorbance at 450 and $540 \mathrm{~nm}$, respectively, using a plate reader (Molecular Devices, LLC).

RNA extraction and Taqman multiplex PCR assay. Total RNA was extracted from UVB-irradiated HaCaT cells, using TRIzol reagent according to the manufacturer's protocol (Invitrogen; Thermo Fisher Scientific, Inc.). RT-qPCR was performed using 
TaqMan assays (Applied Biosystems; Thermo Fisher Scientific, Inc.) specific for involucrin, filaggrin, TGF- $\beta$, interleukin (IL)- $1 \beta$, IL- 8 , and tumor necrosis factor (TNF)- $\alpha$ (involucrin; Hs00846307_s1, filaggrin; Hs00856927_g1, TGF- $\beta$; Hs00998133_m1, IL-1 $\beta$; Hs00174097_m1, IL-8; Hs00174103_ $\mathrm{m} 1$ and TNF- $\alpha$; Hs01113624_g1) with a QuantStudio 6 Flex Real-Time PCR system (Applied Biosystems; Thermo Fisher Scientific, Inc.). The thermocycling conditions were as follows: $95^{\circ} \mathrm{C}$ for $10 \mathrm{~min}, 40$ cycles of denaturation at $95^{\circ} \mathrm{C}$ for $15 \mathrm{sec}$, followed by annealing and extension at $60^{\circ} \mathrm{C}$ for $1 \mathrm{~min}$. Each sample was assayed in triplicate and relative mRNA expression levels were calculated using the $\Delta \Delta \mathrm{Cq}$ method (21) and normalized to that of $\beta$-actin in each sample.

Western blotting. To extract total protein, $\mathrm{HaCaT}$ cells were homogenized and lysed in RIPA assay buffer containing a protease inhibitor (ATTO Corporation, Tokyo, Japan). Protein $(15 \mu \mathrm{g})$ was separated by SDS-PAGE on a $10 \%$ polyacrylamide gel and then transferred to a polyvinylidene difluoride membrane, which was then incubated overnight at $4^{\circ} \mathrm{C}$ with the following primary antibodies (all, 1:1,000): Procollagen (cat. no. 84336), pERK (cat. no. 9101), ERK (cat. no. 9102), pMEK (cat. no. 9154), MEK (cat. no. 9126), pp38 (cat. no. 9215), p38 (cat. no. 9212), pJNK (cat. no. 9251), JNK (cat. no. 9252), and $\beta$-actin (cat. no. 4970). The membrane was washed three times for 10 min each with PBS containing $0.1 \%$ tween 20 , which was followed by incubation for $2 \mathrm{~h}$ at room temperature with appropriate horseradish peroxidase conjugated anti-rabbit secondary antibodies (1:10,000; cat. no. 7074; Cell signaling Technology, Inc.). Protein bands were visualized using an enhanced chemiluminescence kit (Thermo Fisher Scientific, Inc.) and analyzed using ImageQuant LAS 4000 (GE Healthcare).

Statistics. Measurements were performed in triplicate and all data are presented as the mean \pm standard error of the mean. The significance of differences between groups was evaluated by performing an analysis of variance with Tukey's multiple comparisons test with GraphPad Prism version 7.03 software (GraphPad Software, Inc.). P $<0.05$ was considered to indicate a statistically significant difference.

\section{Results}

ACT 3302 increases cell viability and protects against UVB-induced cell damage in keratinocytes. The effect of ACT 3302 on $\mathrm{HaCaT}$ cell proliferation following exposure to UVB was investigated first. Cell viability was reduced to $49.4 \%$ of normal levels by UVB irradiation. In cells preincubated with $1 \times 10^{8}$ ACT 3302 cells, cell viability was $81.4 \%$ of normal levels following irradiation in dose-dependent manner and this difference was significant $(\mathrm{P}<0.001 ;$ Fig. 1$)$.

Effect of ACT 3302 on UVB-induced secretion of skin hydration factors. The effect of ACT 3302 on UVB-induced secretion of skin hydration factors, namely epidermal ECM components was also determined. UVB irradiation decreased HA levels, as determined by ELISA (Fig. 2A), indicating that ECM breakdown induced by UVB radiation contributes to skin aging. HA levels were increased significantly $(\mathrm{P}<0.001)$

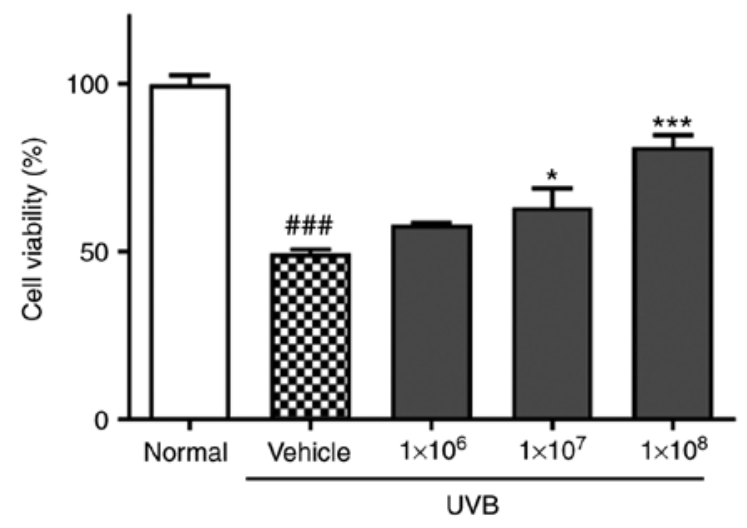

Figure 1. Viability of human keratinocytes following UVB exposure. $\mathrm{HaCaT}$ cells were pretreated with various concentrations of Lactobacillus acidophilus IDCC 3302 for $24 \mathrm{~h}$ and then irradiated. The normal group is the vehicle group with no UVB treatment. Data are presented as percentages relative to the normal group. ${ }^{\# \#} \mathrm{P}<0.001$ vs. the normal group; ${ }^{*} \mathrm{P}<0.05$ and ${ }^{* * * *} \mathrm{P}<0.001$ vs. the UVB vehicle group. UVB, ultraviolet B.

in a dose dependent manner. In addition, it was demonstrated that the expression of involucrin, filaggrin, and TGF- $\beta$ was decreased by UVB. The mRNA expression of involucrin, filaggrin, and TGF- $\beta$ in UVB-induced cells treated with ACT 3302 increased in a dose-dependent manner, as compared to that in UVB-exposed cells (Fig. 2B-D).

ACT 3302 protects HaCaT cells from oxidative stress and suppresses inflammation in response to UVB radiation. To determine whether ACT 3302 can promote radical scavenging, the activities of antioxidant enzymes in $\mathrm{HaCaT}$ cells exposed to UVB were examined. SOD activity in vehicle group was reduced compared with the untreated normal group ( 0.53 vs. $4.96 \mathrm{U} / \mathrm{ml}$; Fig. 3A). Also, CAT was significantly reduced in UVB-induced cells compared with normal cells $(\mathrm{P}<0.01$; Fig. 3B). However, SOD and CAT activity were enhanced by ACT 3302 but SOD was saturated at $1 \times 10^{8}$ of ACT 3302 . Therefore, ACT 3302 stimulates the activity of antioxidant enzymes that scavenge free radicals and thereby prevents UVB-induced oxidative stress damage. It was also demonstrated that mRNA levels of the pro-inflammatory cytokines IL-1 $\beta$, IL-8, and TNF- $\alpha$ were significantly upregulated in keratinocyte cells upon UVB exposure $(\mathrm{P}<0.05)$, which was reversed by ACT 3302 treatment (Fig. 4). This suggests that ACT 3302 exerts a protective effect by reducing the inflammatory response to UVB irradiation.

ACT 3302 prevents UVB-induced damage to keratinocytes by inhibiting MAPK signaling. To clarify the mechanism through which ACT 3302 protects keratinocytes from UVB-induced damage, MMP levels in the culture supernatant were detected by ELISA following UVB irradiation. UVB irradiation significantly increased MMP-1, MMP-2, and MMP-9 levels in HaCaT cells $(\mathrm{P}<0.001)$, but this effect was significantly abolished by pre-treatment with ACT 3302 in a dose-dependent manner $(\mathrm{P}<0.001$; Fig. 5A-C). Also, ACT 3302 treatment reversed this trend and increased procollagen phosphorylation (Fig. 5D).

Additionally, the increase in p38, JNK, and MAPK/ERK phosphorylation induced by UVB irradiation was significantly 

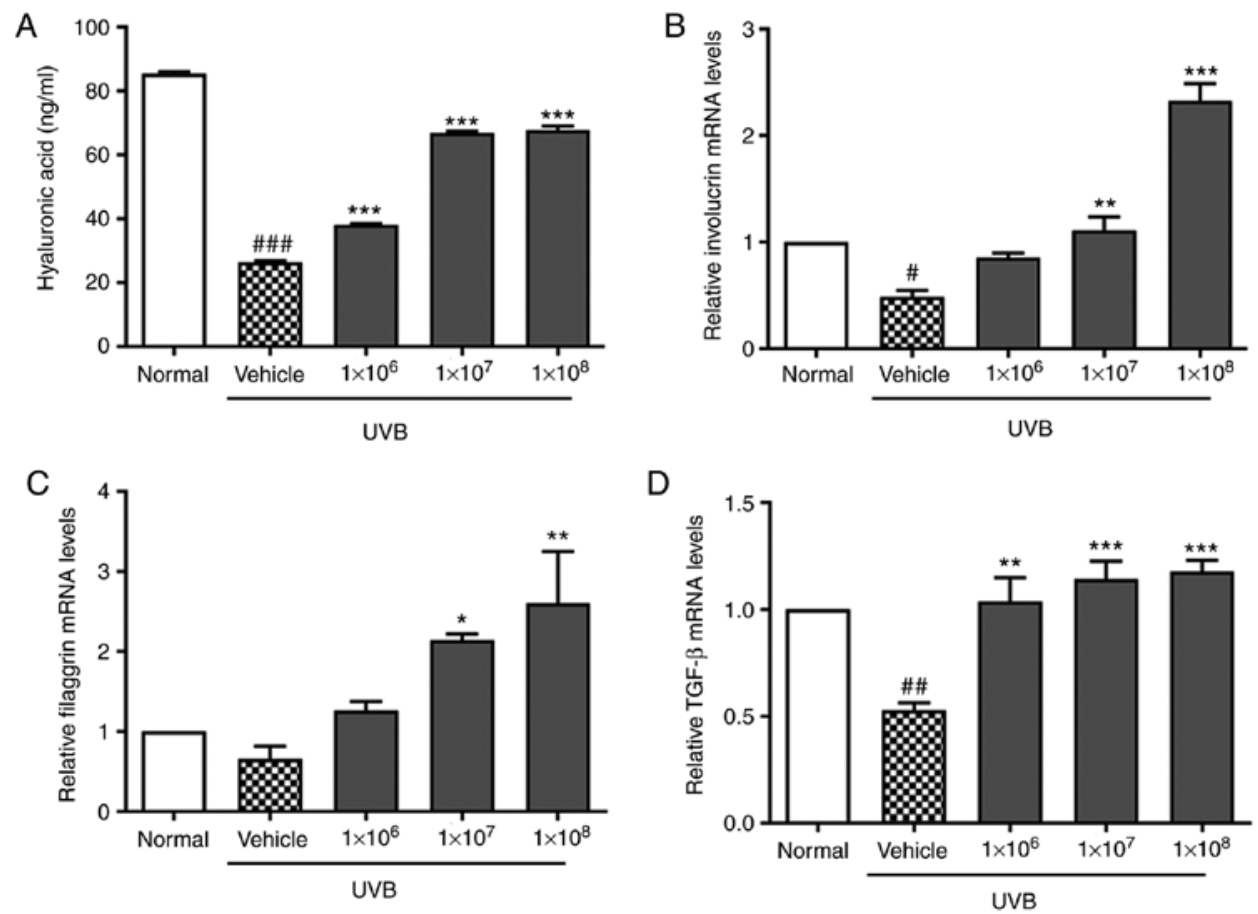

Figure 2. Effects of Lactobacillus acidophilus IDCC 3302 on skin hydration factors. (A) HA levels in UVB-induced HaCaT cells. (B) Involucrin, (C) filaggrin and (D) TGF- $\beta$ mRNA expression levels were evaluated by reverse transcription-quantitative polymerase chain reaction. Normal means the vehicle group with no UVB treatment. ${ }^{\#} \mathrm{P}<0.05,{ }^{\# \#} \mathrm{P}<0.01$ and ${ }^{\# \# \#} \mathrm{P}<0.001$ vs. normal group; ${ }^{*} \mathrm{P}<0.05,{ }^{* *} \mathrm{P}<0.01$ and ${ }^{* * *} \mathrm{P}<0.001$ vs. UVB vehicle group. TGF, transforming growth factor; UVB, ultraviolet B; HA, hyaluronan.
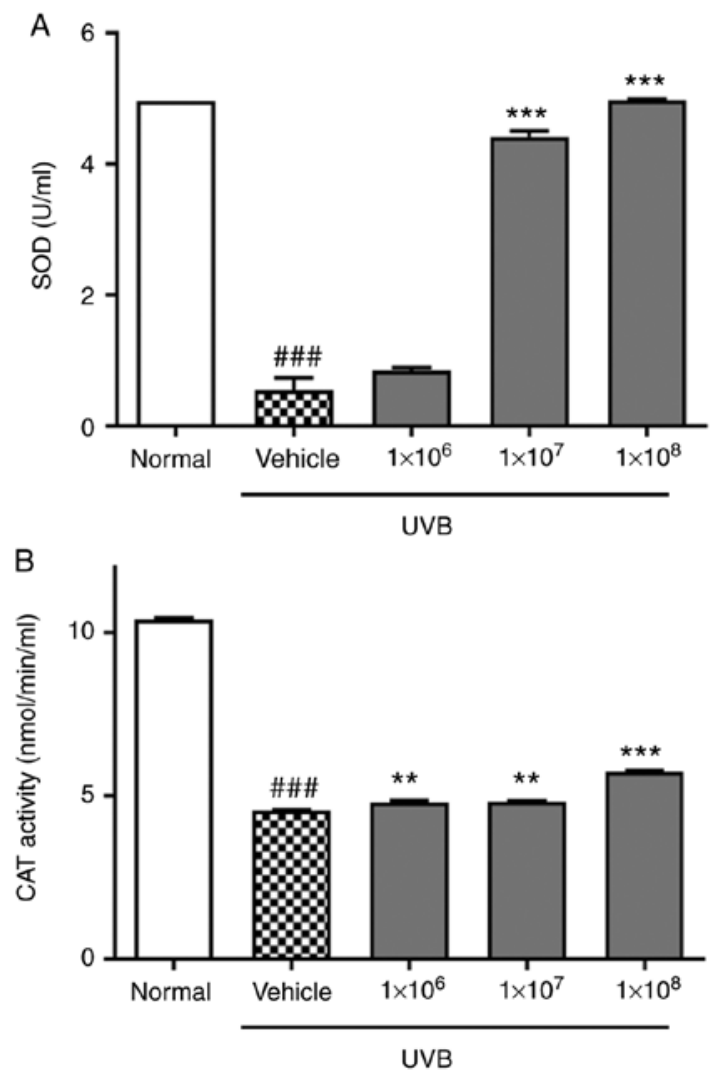

Figure 3. Effects of ACT 3302 on antioxidant enzyme activity in human keratinocytes. (A) SOD and (B) CAT activities were examined in $\mathrm{HaCaT}$ cells exposed to UVB following pretreatment with ACT 3302. Normal means the vehicle group with no UVB treatment. ${ }^{\# \#} \mathrm{P}<0.001$ vs. normal group; ${ }^{* *} \mathrm{P}<0.01$ and ${ }^{* * *} \mathrm{P}<0.001$ vs. UVB vehicle group. SOD, superoxide dismutase; CAT, catalase; UVB, ultraviolet B; ACT 3302, Lactobacillus acidophilus IDCC 3302 . decreased by ACT $3302(\mathrm{P}<0.001)$. These data indicate that ACT 3302 protects $\mathrm{HaCaT}$ cells from UVB-induced damage by inhibiting the activity of ECM-degrading proteins, and this occurs through the modulation of MAPK signaling (Fig. 6).

\section{Discussion}

The present study demonstrated the protective effect of L. acidophilus IDCC 3302 tyndallizate on HaCaT keratinocyte damage induced by UVB exposure. Tyndallizate was analyzed for its nutrients including carbohydrate, crude protein, crude fat, moisture, and ash (22). For small molecule analysis, lactic acid was detected as a major chemical component and it is reported that topical lactic acid has cosmetic benefit (22). In previous studies, beneficial effects of short-chain fatty acids and vitamins produced by probiotics have been reported $(23,24)$. In this study, tyndallizate was hypothesized to be helpful in skin rejuvenation. The detailed mechanisms regarding anti-wrinkle activity require further study.

Skin aging is mainly caused by repeated sun exposure (25). Intrinsically aged skin is characterized by fine wrinkling and reduced elasticity, whereas extrinsically aged skin exhibits deep wrinkles, pigment irregularities, and a substantial loss of elasticity $(26,27)$. Exposure to UV radiation leads to the deposition of abnormal elastin complexes and the denaturation of collagen fibers (28), as well as increased epidermal thickness and alterations in connective tissue organization $(29,30)$. Involucrin is a differentiation marker normally expressed by irreversibly differentiated keratinocytes in the stratum corneum; in a previous study, downregulation of filaggrin was demonstrated to be involved in skin reconstruction in vitro following UVB exposure $(31,32)$. In this study, 

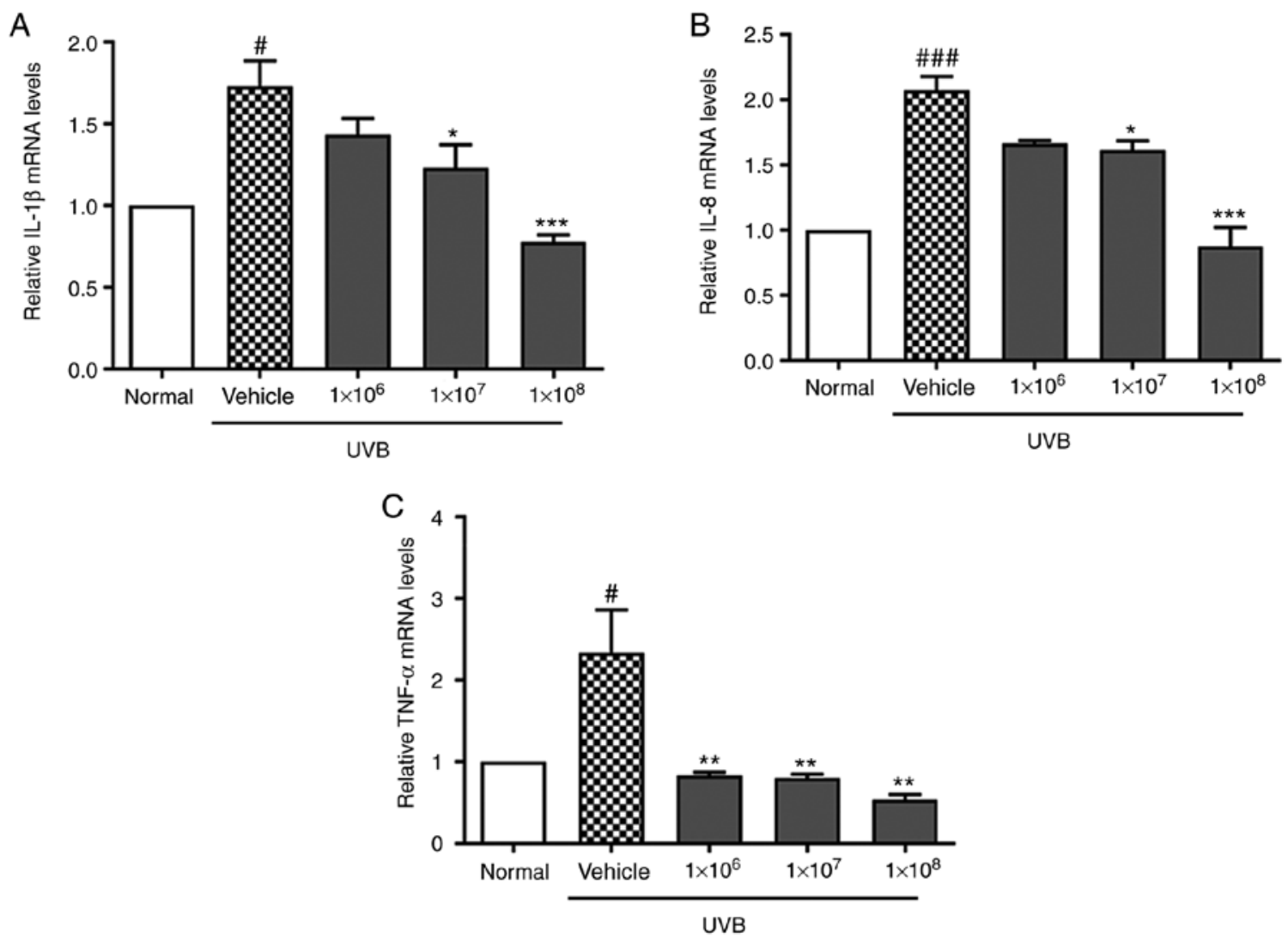

Figure 4. Effects of Lactobacillus acidophilus IDCC 3302 on pro-inflammatory cytokine levels in UVB-irradiated HaCaT cells. (A) IL-1 $\beta$, (B) IL-8 and (C) TNF- $\alpha$ mRNA expression levels were evaluated by reverse transcription-quantitative polymerase chain reaction. Normal means vehicle group with no UVB treatment. ${ }^{\#} \mathrm{P}<0.05$ and ${ }^{\# \# /} \mathrm{P}<0.001$ vs. normal group; ${ }^{*} \mathrm{P}<0.05,{ }^{* * *} \mathrm{P}<0.01$ and ${ }^{* * * *} \mathrm{P}<0.001$ vs. UVB vehicle group. IL, interleukin; TNF, tumor necrosis factor; UVB, ultraviolet B.
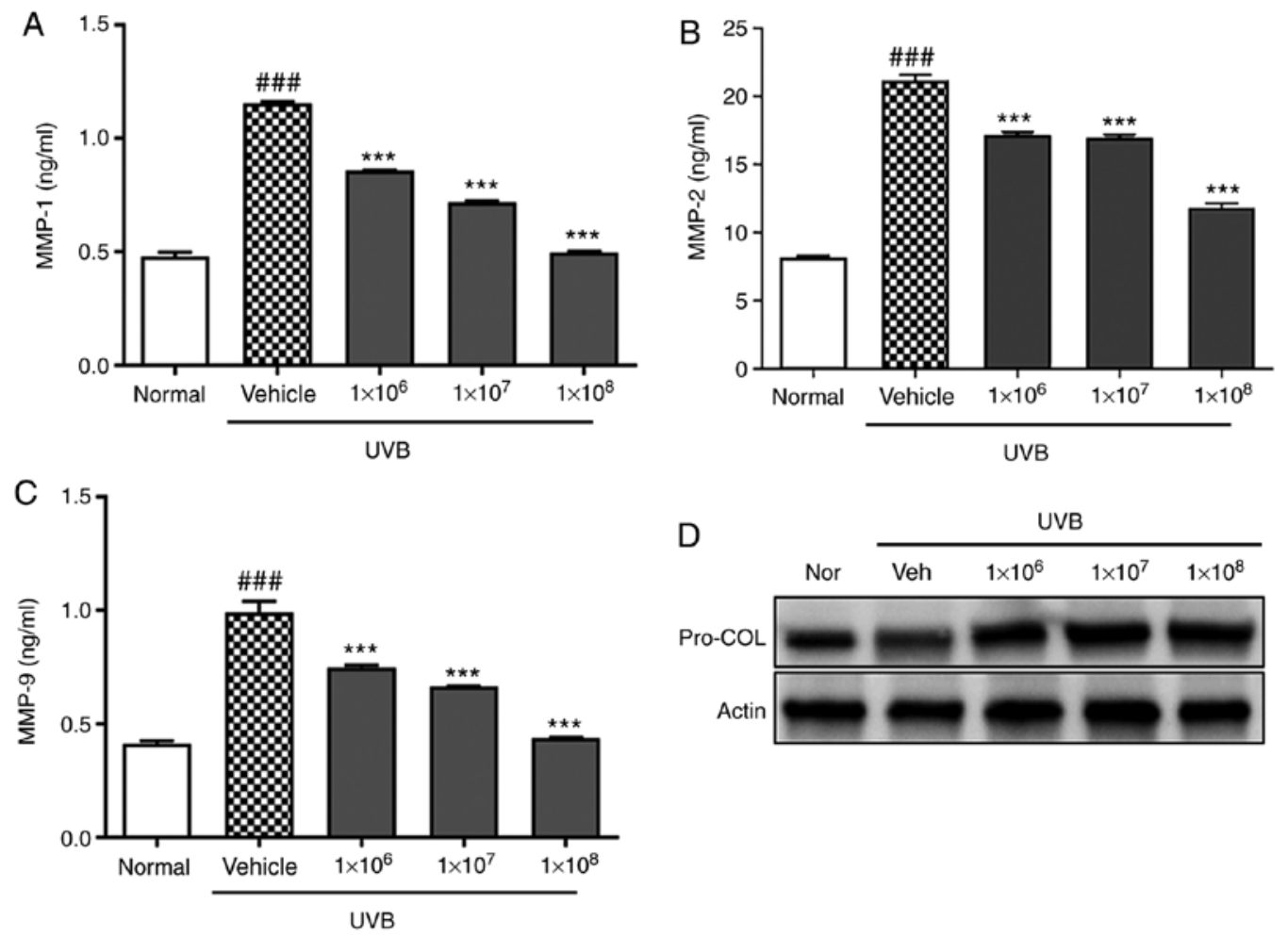

Figure 5. Effects of ACT 3302 on MMP expression and pro-col levels in UVB-irradiated human keratinocytes. HaCaT cells were treated with ACT 3302 for $24 \mathrm{~h}$ before UVB irradiation. (A) MMP-1, (B) MMP-2 and (C) MMP-9 levels in the culture supernatant were detected by ELISA. (D) Pro-col protein levels were determined by western blotting. Normal means the veh group with no UVB treatment. ${ }^{\# \# \#} \mathrm{P}<0.001$ vs. nor group; ${ }^{* * *} \mathrm{P}<0.001$ vs. UVB veh group. MMP, matrix metalloproteinase; UVB, ultraviolet B; ACT 3302, Lactobacillus acidophilus IDCC 3302; Nor, normal; Veh, vehicle; pro-col, pro-collagen. 

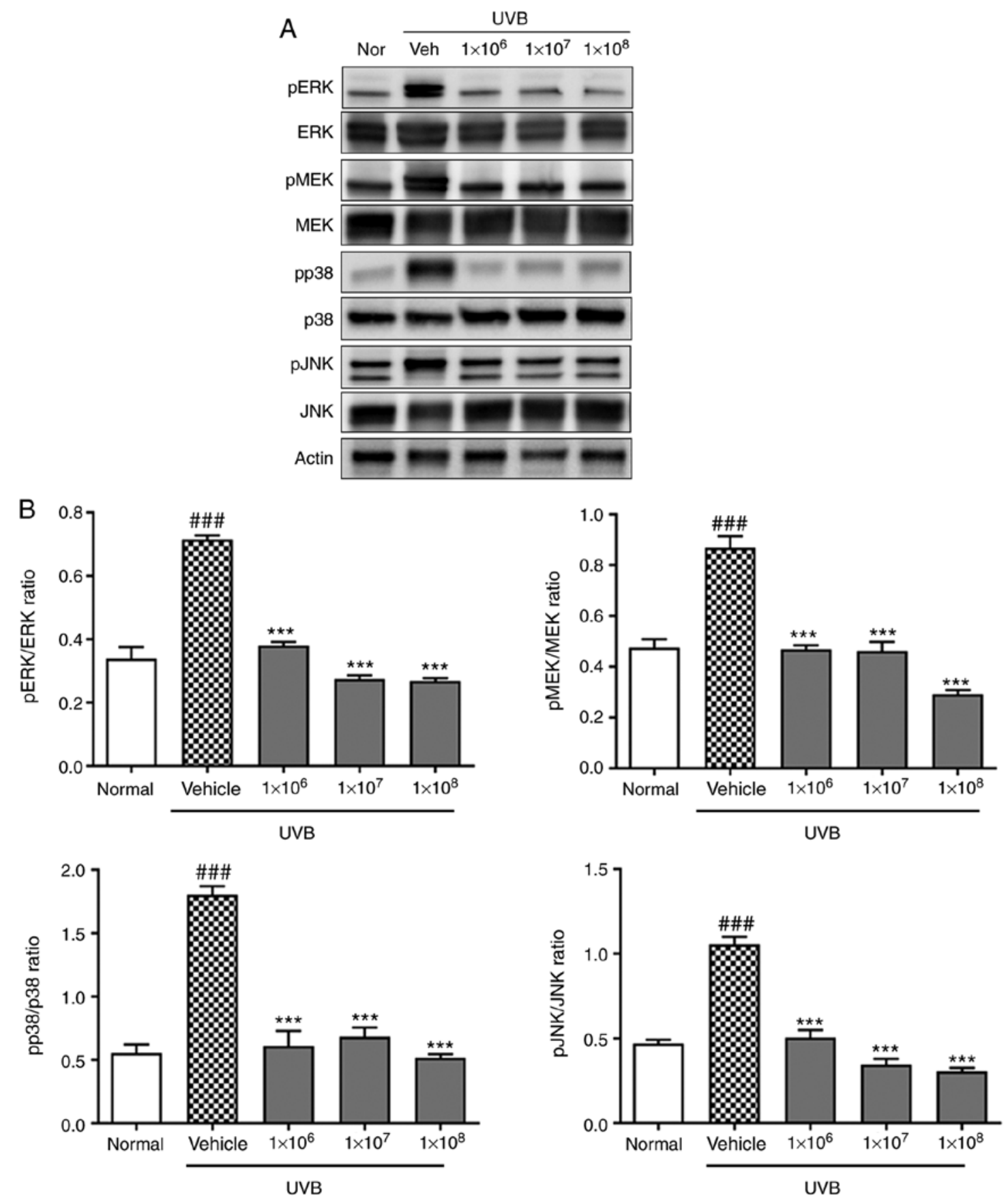

Figure 6. Effects of ACT 3302 on ERK, MEK, p38 and JNK phosphorylation in the UVB-irradiated HaCaT cells. (A) ACT 3302 inhibited the phosphorylation of ERK, MEK, p38 and JNK as determined by western blotting. (B) Relative intensity of data from western blot analysis was analyzed. Normal means the vehicle group with no UVB treatment. ${ }^{\# \# \#} \mathrm{P}<0.001$ vs. normal group; ${ }^{* * * *} \mathrm{P}<0.001 \mathrm{vs.} \mathrm{UVB}$ vehicle group. JNK, c-Jun $\mathrm{N}$-terminal kinase; ERK, extracellular signal regulated kinase; MEK, dual specificity mitogen-activated protein kinase kinase 1; UVB, ultraviolet B; ACT 3303, Lactobacillus acidophilus IDCC 3302.

the upregulation of HA, involucrin, filaggrin, and TGF- $\beta$, an epidermal ECM component that serves an essential role in supporting tissue architecture in the skin and epidermis and protects skin from dryness caused by UVB exposure was observed.

Collagen protects skin from photodamage (33). Type I collagen, which forms the bulk of skin connective tissue in the dermis, is gradually lost during the aging process (34). Exposure to UV radiation can cause collagen breakdown, fragmentation, and disorganization, as well as the inhibition of procollagen biosynthesis (35). In this study, western blot analysis revealed that the downregulation of procollagen expression, relative to that in the normal group, upon UVB irradiation was also reversed by ACT 3302.

Increased activity of MMPs due to chronic sun exposure promotes dermal ECM fragmentation, causing the aged appearance of skin (36). Multiple MMPs including MMP-1 and MMP-9 in the human skin dermis are upregulated during aging, but this is exacerbated by exposure to UV light from the sun (37). In particular, MMP-1 serves an important role in the degradation of dermal collagens in the ECM (38). Indeed, in this study it was observed that MMP-1, MMP-2, and MMP-9 levels were increased in HaCaT cells upon UVB irradiation, it was reversed by ACT 3302. 
ROS are involved in a variety of physiological processes including intracellular signaling, cell proliferation, tumor suppression, immune defense against pathogens, and oxygen homeostasis (39). Following UVB irradiation, SOD and CAT activities were suppressed; however, ACT 3302 stimulated antioxidant enzyme activity and thereby prevented UVB-induced oxidative stress.

Exposing the skin to UV radiation can induce immune suppression and can lead to inflammation (40). In keratinocytes, UVB induces the synthesis of various pro-inflammatory cytokines including TNF- $\alpha$, IL-1, IL-6, IL-8, and IL-10, leading to the activation of nuclear factor- $\kappa \mathrm{B}$ signaling (41). In the present study, UVB irradiation increased IL-1 $\beta$, IL-8, and TNF- $\alpha$ levels in HaCaT cells, it was abolished by ACT 3302.

UVB irradiation induces the production of ROS and activates cell surface receptors, leading to the activation of MAPK signaling, which involves JNK, ERK and p38 (42). MAPK transmits extracellular signals to the nucleus and is one of the most important signal transduction pathways activated by UVB radiation (43). The present study identified that ACT 3302 inhibited the UVB-induced activation of JNK, p38, MEK and ERK.

In conclusion, it was demonstrated that ACT 3302 reduces skin damage caused by UVB radiation by increasing the activity of antioxidant enzymes and skin hydration factors, as well as by suppressing MMP levels along with pro-inflammatory cytokine production through the inhibition of MAPK signaling pathway. The results of the present study provide evidence that probiotics are not only important for maintaining gut health but can have additional (cosmetic) benefits such as preventing skin aging.

\section{Acknowledgements}

Not applicable.

\section{Funding}

The present study was supported by the Korea Institute of Oriental Medicine (grant no. K17300).

\section{Availability of data and materials}

All data generated or analyzed during the present study are available upon reasonable request.

\section{Authors' contributions}

AI, BL, DK and SC conceived and designed the current study, and acquired the data. $\mathrm{AI}$ and $\mathrm{BL}$ performed the experiments and analyzed the data. SC revised and gave final approval of the current version to be published. All authors read and approved the final manuscript.

\section{Ethics approval and consent to participate}

Not applicable.

\section{Patient consent for publication}

Not applicable.

\section{Competing interests}

The authors declare that they have no competing interests.

\section{References}

1. Jenkins G: Molecular mechanisms of skin ageing. Mech Ageing Dev 123: 801-810, 2002.

2. Kammeyer A and Luiten RM: Oxidation events and skin aging. Ageing Res Rev 21: 16-29, 2015.

3. D'Orazio J, Jarrett S, Amaro-Ortiz A and Scott T: UV radiation and the skin. Int J Mol Sci 14: 12222-12248, 2013.

4. Rittié L and Fisher GJ: UV-light-induced signal cascades and skin aging. Ageing Res Rev 1: 705-720, 2002.

5. Lim SH, Kim SM, Lee YW, Ahn KJ and Choe YB: Change of biophysical properties of the skin caused by ultraviolet radiation-induced photodamage in Koreans. Skin Res Technol 14: 93-102, 2008.

6. Dai G, Freudenberger T, Zipper P, Melchior A, Grether-Beck S, Rabausch B, de Groot J, Twarock S, Hanenberg H, Homey B, et al: Chronic ultraviolet B irradiation causes loss of hyaluronic acid from mouse dermis because of down-regulation of hyaluronic acid synthases. Am J Pathol 171: 1451-1461, 2007.

7. Steinert PM and Marekov LN: The proteins elafin, filaggrin, keratin intermediate filaments, loricrin, and small proline-rich proteins 1 and 2 are isodipeptide cross-linked components of the human epidermal cornified cell envelope. J Biol Chem 270: 17702-17711, 1995.

8. Bosset S, Bonnet-Duquennoy M, Barré P, Charlon A, Kurfurst R, Bonté F, Schnébert S, Le Varlet B and Nicolas JF: Photoageing shows histological features of chronic skin inflammation without clinical and molecular abnormalities. Br J Dermatol 149: 826-835, 2003.

9. Fligiel SE, Varani J, Datta SC, Kang S, Fisher GJ and Voorhees JJ: Collagen degradation in aged/photodamaged skin in vivo and after exposure to matrix metalloproteinase-1 in vitro. J Invest Dermatol 120: 842-848, 2003.

10. Liebel F, Kaur S, Ruvolo E, Kollias N and Southall MD: Irradiation of skin with visible light induces reactive oxygen species and matrix-degrading enzymes. J Invest Dermatol 132: 1901-1907, 2012.

11. Rinnerthaler M, Bischof J, Streubel MK, Trost A and Richter K: Oxidative stress in aging human skin. Biomolecules 5: 545-589, 2015.

12. Pillai S, Oresajo $\mathrm{C}$ and Hayward J: Ultraviolet radiation and skin aging: Roles of reactive oxygen species, inflammation and protease activation, and strategies for prevention of inflammation-induced matrix degradation-a review. Int J Cosmet Sci 27: 17-34, 2005.

13. Bickers DR and Athar M: Oxidative stress in the pathogenesis of skin disease. J Invest Dermatol 126: 2565-2575, 2006.

14. Davis RJ: The mitogen-activated protein kinase signal transduction pathway. J Biol Chem 268: 14553-14556, 1993.

15. Sun S, Jiang P, Su W, Xiang Y, Li J, Zeng L and Yang S: Wild chrysanthemum extract prevents UVB radiation-induced acute cell death and photoaging. Cytotechnology 68: 229-240, 2016.

16. Adams CA: The probiotic paradox: Live and dead cells are biological response modifiers. Nutr Res Rev 23: 37-46, 2010.

17. Liu Z, Liu W, Ran C, Hu J and Zhou Z: Abrupt suspension of probiotics administration may increase host pathgen susceptibility by inducing gut dysbiosis. Sci Rep 6: 23214, 2016.

18. Gerritsen J, Smidt H, Rijkers GT and de Vos WM: Intestinal microbiota in human health and disease: The impact of probiotics. Genes Nutr 6: 209-240, 2011.

19. Satoh T, Murata M, Iwabuchi N, Odamaki T, Wakabayashi H, Yamauchi K, Abe F and Xiao JZ: Effect of Bifidobacterium breve B-3 on skin photoaging induced by chronic UV irradiation in mice. Benef Microbes 6: 497-504, 2015.

20. Bouilly-Gauthier D, Jeannes C, Maubert Y, Duteil L, Queille-Roussel C,Piccardi N,Montastier C,ManissierP,Piérard G and Ortonne JP: Clinical evidence of benefits of a dietary supplement containing probiotic and carotenoids on ultraviolet-induced skin damage. Br J Dermatol 163: 536-543, 2010.

21. Livak KJ and Schmittgen TD: Analysis of relative gene expression data using real-time quantitative PCR and the 2(-Delta Delta C(T)) method. Methods 25: 402-408, 2001.

22. Smith WP: Epidermal and dermal effects of topical lactic acid. J Am Acad Dermatol 35: 388-391, 1996. 
23. LeBlanc JG, Chain F, Martín R, Bermúdez-Humarán LG, Courau S and Lagella P: Beneficial effects on host energy metabolism of short-chin fatty acids and vitamins produced by commensal and probiotic bacteria. Microb Cell Fact 16: 79, 2017.

24. Preidis GA, Hill C, Guerrant RL, Ramakrishna BS, Tannock GW and Versalovic J: Probiotics, enteric and diarrheal disease, and global health. Gastroenterology 140: 8-14, 2011.

25. Khavkin J and Ellis DA: Aging skin: Histology, physiology, and pathology. Facial Plast Surg Clin North Am 19: 229-234, 2011.

26. El-Domyati M, Attia S, Saleh F, Brown D, Birk DE, Gasparro F, Ahmad $\mathrm{H}$ and Uitto J: Intrinsic aging vs. photoaging: A comparative histopathological, immunohistochemical, and ultrastructural study of skin. Exp Dermatol 11: 398-405, 2002.

27. Tobin DJ: Introduction to skin aging. J Tissue Viability 26 37-46, 2017.

28. Scharffetter-Kochanek K, Brenneisen P, Wenk J, Herrmann G, Ma W, Kuhr L, Meewes C and Wlaschek M: Photoaging of the skin from phenotype to mechanisms. Exp Gerontol 35: 307-316, 2000

29. Chung JH, Seo JY, Lee MK, Eun HC, Lee JH, Kang S, Fisher GJ and Voorhees JJ: Ultraviolet modulation of human macrophage metalloelastase in human skin in vivo. J Invest Dermatol 119 507-512, 2002

30. Sherratt MJ: Tissue elasticity and the ageing elastic fibre. Age (Dordr) 31: 305-325, 2009.

31. Bosset S, Bonnet-Duquennoy M, Barré P, Chalon A, Lazou K, Kurfurst R, Bonté F, Schnébert S, Disant F, Le Varlet B, et al: Decreased expression of keratinocyte betal integrins in chronically sun-exposed skin in vivo. Br J Dermatol 148: 770-778, 2003.

32. Bernerd F and Asselineau D: Successive alteration and recovery of epidermal differentiation and morphogenesis after specific UVB-damages in skin reconstructed in vitro. Dev Biol 183: $123-138,1997$.
33. Chung JH, Seo JY, Choi HR, Lee MK, Youn CS, Rhie G, Cho KH, Kim KH, Park KC and Eun HC: Modulation of skin collagen metabolism in aged and photoaged human skin in vivo. J Invest Dermatol 117: 1218-1224, 2001.

34. Calleja-Agius J, Brincat M and Borg M: Skin connective tissue and ageing. Best Pract Res Clin Obstet Gynaecol 27: 727-740, 2013.

35. Quan T, Qin Z, Xia W, Shao Y, Voorhees JJ and Fisher GJ Matrix-degrading metalloproteinases in photoaging. J Investig Dermatol Symp Proc 14: 20-24, 2009.

36. Yaar M and Gilchrest BA: Photoageing: Mechanism, prevention and therapy. Br J Dermatol 157: 874-887, 2007.

37. Philips N, Auler S, Hugo R and Gonzalez S: Beneficial regulation of matrix metalloproteinases for skin health. Enzyme Res 2011: 427285, 2011.

38. Kähäri VM and Saarialho-Kere U: Matrix metalloproteinases in skin. Exp Dermatol 6: 199-213, 1997.

39. Poljšak $B$ and Dahmane R: Free radicals and extrinsic skin aging. Dermatol Res Pract 2012: 135206, 2012.

40. Clydesdale GJ, Dandie GW and Muller HK: Ultraviolet light induced injury: Immunological and inflammatory effects. Immunol Cell Biol 79: 547-568, 2001.

41. Kirnbauer R, Köck A, Neuner P, Förster E, Krutmann J, Urbanski A, Schauer E, Ansel JC, Schwarz T and Luger TA: Regulation of epidermal cell interleukin- 6 production by UV light and corticosteroids. J Invest Dermatol 96: 484-489, 1991.

42. Roux PP and Blenis J: ERK and p38 MAPK-activated protein kinases: A family of protein kinases with diverse biological functions. Microbiol Mol Biol Rev 68: 320-344, 2004.

43. López-Camarillo C, Ocampo EA, Casamichana ML, Pérez-Plasencia C, Alvarez-Sánchez E and Marchat LA: Protein kinases and transcription factors activation in response to UV-radiation of skin: Implications for carcinogenesis. Int J Mol Sci 13: 142-172, 2012. 